\title{
IMAGE-GUIDED TREATMENT USING AN X-RAY THERAPY UNIT AND GOLD NANOPARTICLES: TEST OF CONCEPT
}

\author{
Cindy Le Loirec*, Dominique Chambellan and David Tisseur \\ CEA, LIST, F-91191 Gif-sur-Yvette, France \\ *Corresponding author: cindy.le-loirec@cea.fr
}

\begin{abstract}
Gold nanoparticles (GNPs) have the potential to enhance the radiation dose locally in conjunction with $\mathrm{kV}$ X-rays used for radiation therapy. As for other radiotherapy modalities, the absorbed dose needs to be controlled. To do that, it is an advantage to know the distribution of GNPs. However, no effective imaging tool exists to determine the GNP distribution in vivo. Various approaches have been proposed to determine the concentration of GNPs and its distribution in a tumour and in other organs and tissues. X-ray fluorescence computed tomography (XFCT) is a promising imaging technique to do that. A new experimental device based on the XFCT technique allowing the in vivo control of GNP radiotherapy treatments is proposed. As a test of concept, experimental acquisitions and Monte Carlo simulations were performed to determine the performance that a XFCT detector has to fulfil.
\end{abstract}

\section{INTRODUCTION}

Contrast agents with high atomic number, such as gold nanoparticles (GNPs), have the potential to enhance the radiation dose locally in external radiotherapy with $\mathrm{kV} \mathrm{X}$-rays. In the kilovoltage region, gold has an attenuation coefficient two orders of magnitude greater than that of soft tissue. So, even a small amount of GNPs allows a dose enhancement inside the tumour volume by sparing surrounding normal tissue provided that the GNP concentration is lower there. The greatest ratio between the absorption coefficient of gold and that of soft tissue is found near $40-50 \mathrm{keV}$. However, the use of such energies for treatment would be complicated by the high absorption in tissue. Therefore, X-rays of higher energies, near the gold K-edge $(80.7 \mathrm{keV})$, are usually used. Several developments have already been performed with monoenergetic beams around the gold K-edge, using synchrotron radiation, but that technique is not useful in a clinical context. Instead, more traditional $\mathrm{X}$-ray production methods are considered with some refinements in the filtration to optimise the photon energy distribution of the beam ${ }^{(1)}$.

One of the key tasks during preclinical studies of such a treatment method is to determine the biodistribution of GNPs injected into animals. Indeed, the $K_{\alpha}$ fluorescent X-ray energies of gold (66.99 and 68.80 $\mathrm{keV}$ ) are both able to escape a body when produced by polychromatic X-rays ${ }^{(2,3)}$. However, it has been noted $^{(4,5)}$ that the following three technical challenges are yet to be met satisfactory to perform in vivo quantification of GNPs:

- Goal 1: imaging of GNPs distributed within the tumour and critical organs,

- Goal 2: quantification of the amount of GNPs present,
- Goal 3: achieving system sensitivity capable of detecting GNP concentration typically found in tumours.

X-ray fluorescence computed tomography (XFCT) has shown the potential to overcome these challenges by providing GNP images as well as concentration information $^{(4,6-8)}$. XFCT imaging of GNP-loaded phantoms has been performed using $\mathrm{K} X$-ray fluorescence $(\mathrm{K}-\mathrm{XRF})$ in gold $^{(4)}$. However, relatively high GNP concentrations were tested by the system. Simulation studies comparing X-ray fluorescence CT and K-edge CT show that fluorescence imaging can provide a better sensitivity for very low tracer concentration $^{(9)}$.

The development of a new experimental device based on the XFCT technique and allowing in vivo control of GNP radiotherapy treatments is now proposed. As a test of concept, in a first step the X-ray tube and the detector used at the authors' institute for non-destructive testing were used. In parallel, Monte Carlo (MC) simulations performed with PENELOPE were used; on one hand, as an optimisation tool to enhance the experimental set-up, and on the other hand, as a planning system to estimate the GNP concentration needed to treat tumours.

\section{MATERIALS AND METHODS}

\section{Experimental set-up}

The proposed system (Figure 1) was configured according to the experimental set-up reported by MacMahon et al. ${ }^{(10)}$ and consisted of a polychromatic X-ray source $(125 \mathrm{kVp}, 300 \mu \mathrm{A})$, a $6 \mathrm{~mm}$ diameter collimator, a spectrometer system (XR 100T-CdTe, Amptek, Inc., USA), a multichannel analyser (Novelec SM-1024) and a phantom. 

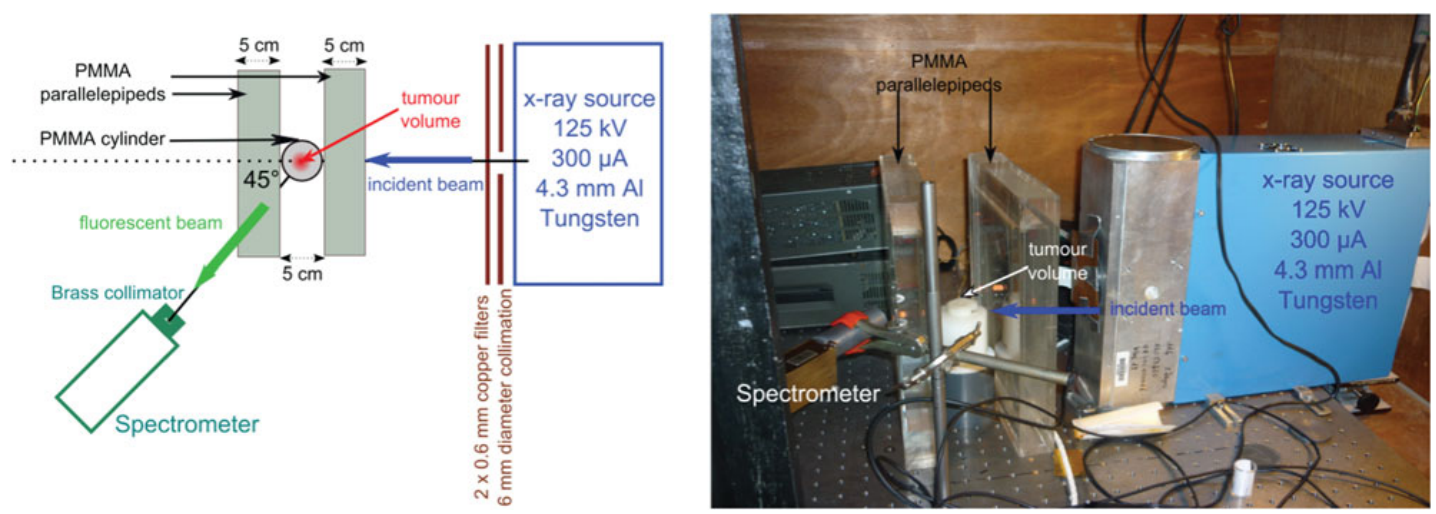

Figure 1. Experimental set-up: the tumour volume is placed on the PMMA cylinder and between the two PMMA inserts and the incident beam is impinging perpendicularly on them. The spectrometer is placed at $135^{\circ}$ from the incident beam.

The XR 100T-CdTe was a thermoelectrically cooled $5 \times 5 \times 1 \mathrm{~mm}^{3}$ CdTe diode X-ray detector and preamplifier. Its energy resolution was $\sim 1.5 \mathrm{keV}$ (FWHM) at $150 \mathrm{keV}$ and $0.6 \mathrm{keV}$ at $59.54 \mathrm{keV}$ $\left({ }^{241} \mathrm{Am}\right.$ source). It had high detection efficiency up to $100 \mathrm{keV}$, which made it ideal for XRF applications. Its calibration was performed with a metallic sheet of pure gold $(99 \%)$.

The phantom was composed of two PMMA parallelepipeds of $5 \mathrm{~cm}$ thickness mimicking the healthy tissue and a cylindrical 'tumour' volume of $5 \mathrm{~cm}$ diameter, positioned between the two PMMA parallelepipeds and in the intersection of the incident and detected beams. The tumour volume was made of PMMA. It had a small hole into which a sheet of pure gold of $1 \mathrm{~mm}$ height and $25 \mathrm{~mm}$ diameter was inserted. The dimensions of the sheet were chosen to obtain a concentration of gold in the tumour volume equivalent to $10 \mathrm{mg} \mathrm{Au} \mathrm{g}^{-1}$ of tumour.

Several strategies were applied to effectively minimise the unwanted background in the registered pulseheight distributions: (i) two 0.6-mm-thick copper filters were placed at the exit of the collimated beam to reduce the unnecessary $\mathrm{X}$-rays with energies below gold K-edge energy $(80.7 \mathrm{keV})$, (ii) the spectrometer was positioned at $135^{\circ}$ to the incident X-ray beam to minimise the impact of the tungsten peaks and of the Compton scattered X-rays in the region of the $\mathrm{Au}$ peaks, and (iii) a brass collimator with an opening of 6 $\mathrm{mm}$ diameter was placed to cover the sensitive element of the spectrometer in order to receive the directional beam while improving the signal-to-noise ratio.

A first measurement was acquired with gold and without any PMMA phantom to detect the gold peaks and to calibrate the spectrometer's energy scale. A second measurement was performed with the PMMA inserts and the tumour volume without gold. A third measurement was performed with the PMMA inserts and the tumour cylindrical volume with gold. The three measurements lasted $10 \mathrm{~min}$ at $300 \mu \mathrm{A}$.

\section{MC simulations}

Simulations were performed with the 2006 version of PENELOPE $^{(11)}$. An RQR10 spectrum $^{(12,13)}$ was simulated with a $0.95^{\circ}$ aperture. The simulated geometry corresponded to the experimental set-up. A brass collimator with a $6 \mathrm{~mm}$ diameter opening was placed on the beam axis, and all the photons emerging it were registered to build an energy spectrum. The same brass collimator was also positioned at $45^{\circ}$ from the beam axis, and the photons emerging it were also registered.

A second simulation was performed with the same parameters, except that the tumour cylinder was replaced by water.

\section{RESULTS}

\section{Experimental results}

Figure 2 shows the spectra obtained with and without any gold sheet in the tumour volume. Both spectra present a Compton scattering front between 62 and 66 $\mathrm{keV}$. The $K_{\beta}$ peaks of tungsten are visible at 67.2 and $69.1 \mathrm{keV}$ for the 'PMMA spectrum' and the $\mathrm{Au} K_{\alpha}$ peaks can be detected at 67.0 and $68.8 \mathrm{keV}$ for the 'Gold + PMMA spectrum'. The difference between both spectra is plotted in the second panel of Figure 2. $\mathrm{Au}$ peaks are hardly detected due to the high background level.

\section{Simulated results}

Spectra simulated with and without gold in the tumour volume are reported in Figure 3. The difference between the two simulated spectra is shown in 

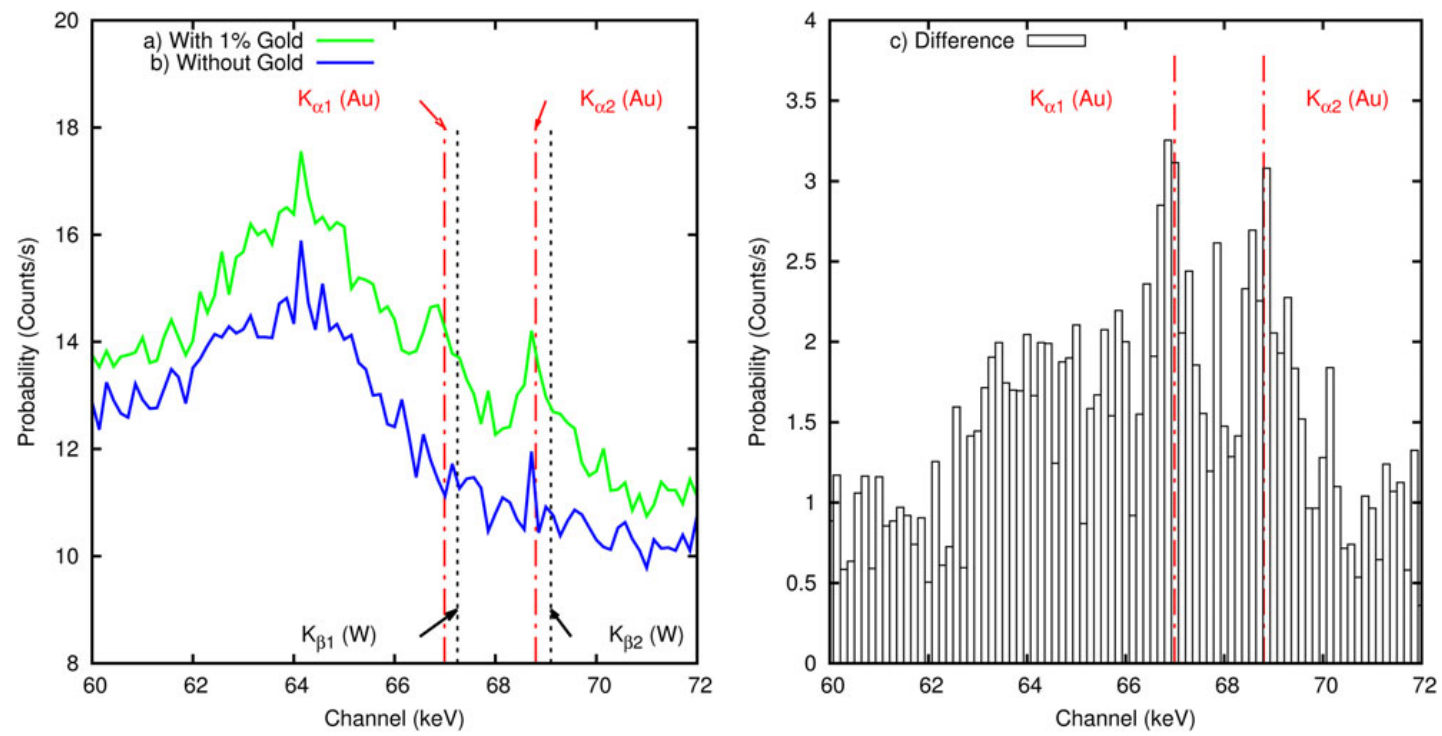

Figure 2. In the first panel, experimental spectra obtained with (a) and without (b) gold inserted into the small cylindrical phantom are plotted. In the second panel, the difference between the two spectra is plotted. The dashed and dotted lines indicate the $K_{\alpha} \mathrm{X}$-ray energies of gold (66.99 and $\left.68.80 \mathrm{keV}\right)$ and the dotted lines the $K_{\beta} \mathrm{X}$-ray energies of tungsten $(67.244$ and $69.1 \mathrm{keV})$.
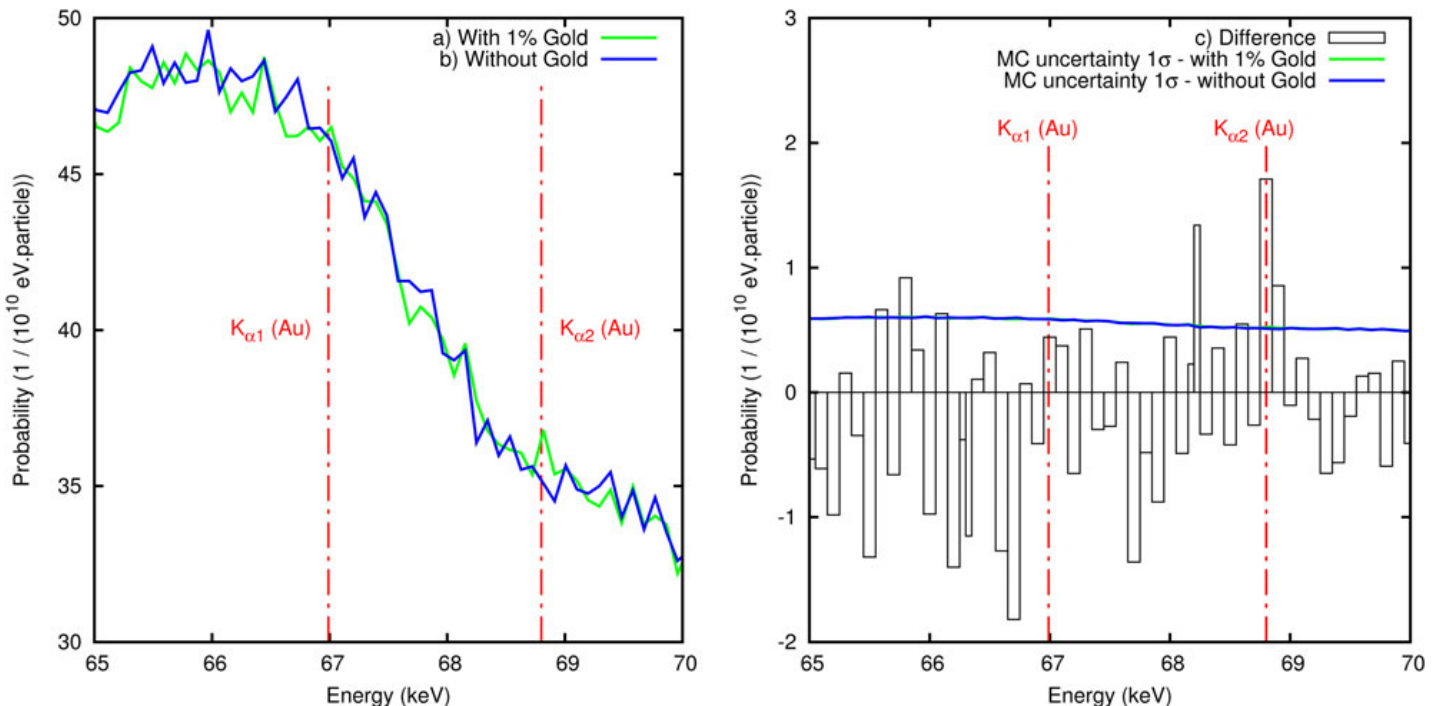

Figure 3. Simulated spectra obtained with (a) and without gold (b) inserted into the small cylindrical phantom. In the second panel, the difference between the two spectra is plotted. The dashed and dotted lines indicate the $K_{\alpha}$ fluorescent X-ray energies of gold (66.99 and $68.80 \mathrm{keV})$.

the second panel of Figure 3. It shows a very good discrimination of the Au $K_{\alpha 2}$ peak, due to the better energy resolution used in the MC simulation and to the absence of background induced by the detector. However, the $\mathrm{Au} K_{\alpha 1}$ peak is hardly detectable, probably being inferior due to the MC uncertainty.

\section{DISCUSSION}

This pilot study has been performed for one experimental and one simulated configuration. The results show that gold peaks are not easily detected because their contribution is mixed with tungsten $K_{\alpha}$ peaks, 
phantom Compton scattering and interactions in the detector. It has been shown ${ }^{(10)}$ that an optimisation of the beam filtration can be performed to optimise the spectral shape of the excited source and increase the sensitivity. Moreover, the detector position has also to be tested in order to find the most suitable detection set-up. Scattered X-rays can be minimised by using a backscatter detector orientation ${ }^{(14)}$. Further simulations will be performed to determine the experimental set-up that is most suitable for application in the present study. To improve the simulation, the detector model will be integrated into the simulation to analyse its impact on the Au peaks detection.

Moreover, in a first step, the authors' laboratory detector usually used for applications in non-destructive testing was used to check the feasibility of the method. However, the energy resolution of this one is too bad $(1.5 \mathrm{keV}$ at $150 \mathrm{keV})$ to allow a good discrimination of the gold peaks in the Compton background. A better energy resolution is needed to allow a good discrimination of the Au peaks.

In the future, the authors plan to test the performance of a detector initially developed in their institute for Astrophysics $(15,16)$. This is an imaging spectrometer (CALISTE HD), which uses a 1-mm-thick Schottky CdTe detector divided into 256 pixels disposed in a $16 \times 16$ matrix with a pixel pitch of 625 $\mu \mathrm{m}$. It has been tested with a ${ }^{241} \mathrm{Am}$ source, and excellent spectroscopic performance was obtained, in particular between 10 and $100 \mathrm{keV}(0.56$ and $0.67 \mathrm{keV}$ FWHM at 13.9 and $59.5 \mathrm{keV}$, equivalent to 4 and 1.1 $\%$ energy resolution, respectively). A better energy resolution $(\sim 0.52 \mathrm{keV}$ at $59.5 \mathrm{keV})$ can be attained for the present application after a finer calibration step and a reduction of the electronical noise.

\section{CONCLUSION}

Measurements performed during this study prove the feasibility to detect Au peaks in GNP radiotherapy treatments. The MC simulations show that a detector with a better energy resolution than the one used during this study is needed to get a good discrimination of the peaks. Other parameters of the detector have also to be taken into account, such as the calibration process, the scattering interactions into the elements of the detector, the pile-up effect and the dead-time to minimise the background visible in Figure 2. A more detailed MC simulation taking into account the geometry of the detector could provide useful information to characterise the ideal detector. Experimental acquisitions will thus be conducted with a better energy resolution detector developed initially for astrophysical applications. In parallel, MC simulations will be performed to optimise the experimental set-up and quantify the performance of the detector.

\section{REFERENCES}

1. Ricketts, K., Castoldi, A., Guazzoni, C., Ozkan, C., Christodoudou, C., Gibson, A. P. and Royle, G. J. A quantitative $x$-ray detection system for gold nanoparticle tumour biomarkers. Phys. Med. Biol. 57, 5543-5555 (2012).

2. Popovtzer, R., Agrawal, A., Kotov, N. A., Popovtzer, A., Balter, J., Carey, T. E. and Kopelman, R. Targeted gold nanoparticles enable molecular CT imaging of cancer. Nano Letters 8, 4593-4596 (2008).

3. Lee, T. Y. Functional CT: physiological models. Trends Biotechnol. 20(Suppl.), S3-S10 (2002).

4. Cheong, S. K., Jones, B. L., Siddiqi, A. K., Liu, F., Manohar, N. and Cho, S. H. X-ray fluorescence computed tomography (XFCT) imaging of gold nanoparticle-loaded objects using 110 kVp x-rays. Phys. Med. Biol. 55, 647-662 (2010).

5. Ricketts, K., Guazzoni, C., Castoldi, A., Gibson, A. P. and Royle, G. J. An x-ray fluorescence imaging system for gold nanoparticle detection. Phys. Med. Biol. 58, $7841-7855$ (2013).

6. Schlomka, J. P. et al. Experimental feasibility of multienergy photon counting $K$-edge imaging in pre-clinical computed tomography. Phys. Med. Biol. 53, 4031-4047 (2008).

7. Takeda, T., Wu, J., Thet-Thet-Lwin, Huo, Q., Yuasa, T., Hyodo, K., Dilmanian, F. A. and Akatsuka, T. X-ray fluorescent CT imaging of cerebral uptake of stableiodine perfusion agent iodoamphetamine along analog IMP in mice. J. Synchrotron Radiat. 16, 57-62 (2009).

8. Jones, B. and Cho, S. The feasibility of polychromatic cone-beam $x$-ray fluorescence computed tomography (XFCT) imaging of gold nanoparticle-loaded objects: a Monte Carlo study. Phys. Med. Biol. 56, 3719-3730 (2011).

9. Bazalova, M., Kuang, Y., Pratx, G. and Xing, L. Investigation of $x$-ray fluorescence computed tomography (XFCT) and K-edge imaging. IEEE Trans. Med. Imaging 31, 1620-1627 (2012).

10. McMahon, S. J., Mendenhall, M. H., Jain, S. and Currell, F. Radiotherapy in the presence of contrast agents: a general figure of merit and its application to gold nanoparticles. Phys. Med. Biol. 53, 5635-5651 (2008).

11. Salvat, F., Fernández-Varea, J. M. and Sempau, J. PENELOPE-2006, A code system for Monte Carlo simulation of electron and photon transport. OECD ISBN 9264-02301-1, Barcelona, Spain (2006).

12. Oliveira, P. M. C., Squair, P. L., Noguiera, M. S. and da Silva, T. A. Uniformity and field size of filtered $x$-ray beams. In: Proceedings of the 2007 International Nuclear Atlantic Conference - INAC 2007, Santos, ABEN (2007).

13. Oliveira, P. M. C., da Silva, T. A., Baptista, A. T. and Pereira, E. G. On implementing reference radiations for calibrating and testing solid state detectors for dose measurements in radiology. In: Proceedings of the 15th International Conference on Solid State Dosimetry, Delft Technology University (2007).

14. Ahmad, M., Bazalova, M., Xiang, L. and Xing, L. Order of magnitude sensitivity increase in $x$-ray fluorescence computed tomography (XFCT) imaging with an optimized spectro-spatial detector configuration: theory 
and simulation. IEEE Trans. Med. Imaging 33, 16. Dubos, S., Lemaire, H., Schaune, S., Limousin, O., 1119-1128 (2014).

15. Meuris, A. et al. Caliste-SO, a CdTe based spectrometer for bright solar event observations in hard X-rays. Nucl. Instrum. Meth. Phys. Res. A 787, $72-77$ (2015). Carrel, F., Schoepff, V. and Blondel, C. ORIGAMIX, a CdTe-based spectro-imager development for nuclear applications. Nucl. Instrum. Meth. Phys. Res. A 787, 302-307 (2015). 